\title{
Group analysis of structure equations for stars in radiative and convective equilibrium
}

\author{
Marek Szydłowski \\ Astronomical Observatory, Jagiellonian University, \\ Orla 171, 30-244 Kraków, Poland \\ Andrzej J. Maciejewski \\ Torun Centre for Astronomy, Nicolaus Copernicus University, \\ Gagarina 11, 87-100 Toruń, Poland
}

\begin{abstract}
It is proposed to use the Lie group theory of symmetries of differential equations to investigate the system of equations describing a static star in a radiative and convective equilibrium. It is shown that the action of an admissible group induces a certain algebraic structure in the set of all solutions, which can be used to find a family of new solutions. We have demonstrated that, in the most general case, the equations admit an infinite parameter group of quasi-homologous transformations. We have found invariants of the symmetries group which correspond to the fundamental relations describing a physical characteristic of the stars such as the Hertzsprung-Russell diagram or the mass-luminosity relation. In this way we can suggest that group invariants have not only purely mathematical sense, but their forms are closely associated with the basic empirical relations.
\end{abstract}

\section{Introduction}

A modern theory of stars interiors and their evolution was founded on the works of Emden, Lane, Ritter and Kelvin [1. They studied equilibrium configurations of polytropic and isothermic gaseous spheres. The important results were obtained by Rudzki [2] who introduced the notion of homologous transformations. Eddington [3] described the effectiveness of radiation transport of energy in a star and build the standard model of stellar structure. Chandrasekhar [1] formulated the homologous theorem known as the Stromgren theorem. Schwarzschild [4 described the thermonuclear processes as a source of energy of the stars. Some properties of stellar structure can be expressed in terms of the Lie group theory of symmetries to obtain the deeper insight in structure of solution of equation for stars in radiative and connective equilibrium [5, 6].

One of the main problems in the group analysis of differential equations is the investigation of properties of the group admissible by the differential equations structure. In the set of all solutions the action of an admissible group induces a certain algebraic structure which can be used to find a family of new solutions from the known ones [8, 9, 10, 11, 12, 13].

In the present work we apply the Lie group theory to investigate group properties of the system of four structure equations describing Newtonian static stars in a radiative and convective equilibrium. Two of these equations, namely the hydrostatic equilibrium and the mass continuity equation, were investigated by Collins from the point of view of the group theory [14.

As is well known from Stromgren's theorem [15, new solutions, for such a system of equations, can be obtained from the known ones through the homologous transformations. The new solutions describe new configurations with different masses, radii, and chemical compositions (the so-called homologous stars). We generalize this result by introducing the 
notion of quasi-homologous stars, i.e., stars whose equations of state admits quasi-homology symmetries. The homologous stars are a special case of the quasi-homologous ones. At the present stage of our investigation, this should be treated as a purely mathematical result, although it cannot be excluded that the obtained dependencies between luminosity and temperature, mass and temperature, and so on could be employed in a manner similar to that done by Stromgren [15] to fully interpret the Hertzsprung-Russell diagram.

The energy of the star radiated away from its surface is generally replenished from reservoirs situated in the very hot central region. The transport of energy is possible due to existence of non-vanishing temperature gradient in the star. The transfer can occur mainly via radiation, conduction and convection. In any case photons, nuclei, electrons are exchanged between hotter and cooler parts where direction of the energy flow is determined by temperature gradient.

The problem of application of symmetry group in the context of equations coming from astrophysics we considered in our previous papers [5, 6, 7]. This paper is a continuation of new quasi-homologous symmetries which are present in the structural equations for stars with convective transport ${ }^{1}$.

\section{Mathematical background}

In the present work we consider differential equation system of the following form

$$
\frac{d u^{i}}{d x}=f^{i}\left(x, u^{1}, \ldots, u^{m}\right), \quad i=1, \ldots, m .
$$

We consider point transformations generated by the infinitesimal operator

$$
X=\xi\left(x, u^{1}, \ldots, u^{m}\right) \frac{\partial}{\partial x}+\sum_{i=1}^{m} \eta^{i}\left(x, u^{1}, \ldots, u^{m}\right) \frac{\partial}{\partial u^{i}} .
$$

For the infinitesimal operator $X$ there is $m$ independent invariants which are solutions to the following system (if we assume analyticity of a field)

$$
\frac{d x}{\xi\left(x, u^{1}, \ldots, u^{m}\right)}=\frac{d u^{1}}{\eta^{1}\left(x, u^{1}, \ldots, u^{m}\right)}=\cdots=\frac{d u^{m}}{\eta^{m}\left(x, u^{1}, \ldots, u^{m}\right)} .
$$

The point transformation generated by $X$ is called homologous if $\xi=a x$ and $\eta^{i}=g^{i} u^{i}$, where $a, g^{i}(i=1, \ldots, m)$ are constants. Of course, if $X \equiv \lambda^{s}(u) \partial_{s}$ then the finite transformation of symmetry $u \rightarrow \bar{u}$ are given as a solution of equation $d \bar{u}^{s} / d \tau=\lambda^{s}(u)$ with the initial conditions $\bar{u}^{s}(\tau=0)=u^{s}, s=1, \ldots, m$. It can easily be seen that system (11) is similarity invariant in this case (for discussion see [16]).

A natural extension of this special case leads us to the notion of quasi-homologous transformations $\xi=\xi(x), \eta^{i}=\eta^{i}\left(u^{i}\right), i=1, \ldots, m$. System (11) admits the infinitesimal operator (2) if and only if the following condition is satisfied

$$
\frac{\partial \eta^{i}}{\partial x}+\sum_{j=1}^{m}\left(\frac{d u^{j}}{d x} \frac{\partial \eta^{i}}{\partial u^{j}}-\frac{d u^{i}}{d x} \frac{d u^{j}}{d x} \frac{\partial \xi}{\partial x}-\frac{d u^{i}}{d x} \frac{\partial \xi}{\partial x}\right)-X\left(f^{i}\right)=0 .
$$

Condition (44) tells us whether the symmetry operator $X$ is admitted by system (1) [8]. It is easily seen that, in the case of a quasi-homologous transformation, equation (4) assumes form

$$
\frac{d \eta^{i}}{d u^{i}}-\frac{d \xi}{d x}=X\left(\ln f^{i}\right)
$$

Now let us consider the space of independent variable $x$, dependent $u^{\alpha}$ and its first derivatives, say $\left(u^{\alpha}\right)^{\prime}(\alpha=1, \ldots, m)$. The action of the Lie group $G$ of the point transformation

\footnotetext{
${ }^{1}$ Sections 2 and 3 are the repetition of formulas with necessary corrections from Ref. 5 and new formulas (24) and (25) from Ref. 7], which are crucial to the present analysis
} 
in the space $(x, u)$ can be extended to the space $\left(x, u, u^{\prime}\right)$. On the other hand an $s$-th order (ordinary or partial) differential equation $F\left(x, u(x), u^{\prime}(x), \ldots, u^{s}(x)\right)=0$ defines a certain manifold $\mathcal{M}$ in the space $\left(x, u, u^{\prime}, \ldots, u^{s}\right)$. We say that $F$ is invariant with respect to the group $G$, provided that the manifold $\mathcal{M}$ is a fixed point with respect to the $s$-th extension of $G$, i.e., $G^{s}(\mathcal{M})=\mathcal{M}$. In the terms of an infinitesimal operator it means that

$$
\left.X^{s} F\right|_{F=0}=0
$$

where for the case of first order differential equations

$$
\begin{aligned}
X^{s} & =X+\xi_{i}^{\alpha}\left(x, u, u^{\prime}\right) \frac{\partial}{\partial u_{i}^{\alpha}} \\
u_{i}^{\alpha} & =\frac{\partial u^{\alpha}}{\partial x^{i}} \\
\xi_{i}^{\alpha} & =D_{i}\left(\eta^{\alpha}\right)-u_{j}^{\alpha} D_{i}\left(\xi^{j}\right) \\
D_{i} & =\frac{\partial}{\partial x^{i}}+u_{i}^{\alpha} \frac{\partial}{\partial u^{\alpha}} .
\end{aligned}
$$

The prolonged operators, which are generators of symmetry of equations in the space $(x, u)$, form the structure of the Lie algebra of a fundamental group.

The different method of construction of a partial solution of the system without knowledge of fundamental group bases on searching some subgroup which is called a similarity group. For which the finite transformations are

$$
\bar{u}^{i}=a^{i} u^{i}, \quad \bar{x}^{\alpha}=a^{n+\alpha} x^{\alpha}, \quad i=1, \ldots, n, \quad \alpha=1, \ldots, m .
$$

and the Lie algebra is determined by the operator

$$
X_{i} \equiv u^{i} \frac{\partial}{\partial u^{i}}, \quad X_{n+\alpha}=x^{\alpha} \frac{\partial}{\partial x^{\alpha}}
$$

where all coefficients - dilatation coefficient- $a^{i}, \ldots, a^{n+m}$ are positive. It is easy to find such a subgroup because all form

$$
a^{j}=\left(a^{1}\right)^{m_{1} j}\left(a^{2}\right)^{m_{2} j} \ldots\left(a^{k}\right)^{m_{k} j}, \quad j=k+1, \ldots, N .
$$

The invariants of this subgroup are given

$$
\Pi=\left(u^{1}\right)^{\alpha_{1}}\left(u^{2}\right)^{\alpha_{2}} \ldots\left(u^{N-1}\right)^{\alpha_{N-1}}\left(u^{N}\right)^{\alpha_{N}}
$$

where $\alpha_{1}, \ldots \alpha_{N}$ are determine from algebraic equations. There is a strictly connection between the dimensional analysis of equations and describing its fundamental group.

\section{Quasi-homologous transformations of structure equa- tions with radiative transport}

The structure for equation for a Newtonian star are as follow 4

$$
\begin{aligned}
\frac{d p}{d r} & =-\frac{G M \rho}{r^{2}} & & \text { hydrostatic equilibrium } \\
\frac{d M}{d r} & =4 \pi r^{2} \rho & & \text { mass continuity } \\
\frac{d L}{d r} & =4 \pi r^{2} \epsilon(\rho, T) & & \text { thermal equilibrium }
\end{aligned}
$$

either

$$
\frac{d T}{d r}=-\frac{3}{16 \pi a c} \frac{\rho}{r^{2}} \frac{L}{T^{3}} \kappa(\rho, T) \quad \text { radiative equilibrium }
$$


or

$$
\frac{d T}{d r}=\frac{\Gamma_{2}-1}{\Gamma_{2}} \frac{T}{p} \frac{d p}{d r}=\Gamma \frac{T}{p} \frac{d p}{d r} \quad \text { adiabatic convective equilibrium }
$$

where $M$ is the mass within the sphere of radius $r, \rho$ is the density, $p$ is the pressure, $L$ is the luminosity at the surface of the sphere of radius $r, T$ is the temperature, $\epsilon$ is the energy generation rate, $\kappa$ is the opacity, $G$ is the gravitational constant, $c$ is the velocity of light, and $a$ is the Stefan-Boltzmann constant.

First we consider the case of radiative equilibrium. The equation for the energy transport through the stellar material can be written as a condition for the temperature gradient necessary for the required energy flow. It supplies the next basic equation for the stellar structure. Assuming the equation of state in the form $p=p(\rho, T)$ we can rewrite equation (17) in the more convenient form

$$
\frac{d \rho}{d r}=\left(\frac{\partial p}{\partial \rho}\right)^{-1}\left(-G M+\frac{3}{16 \pi a c} \frac{L}{T^{3}} \kappa \frac{\partial p}{\partial T}\right) \frac{\rho}{r^{2}} .
$$

Now, we look for the symmetry transformations of equations (8)-(10) and (12) generated by the operator

$$
X=\xi(r) \frac{\partial}{\partial r}+\eta^{1}(\rho) \frac{\partial}{\partial \rho}+\eta^{2}(M) \frac{\partial}{\partial M}+\eta^{3}(L) \frac{\partial}{\partial L}+\eta^{4}(T) \frac{\partial}{\partial T} .
$$

If we denote

$$
f=-G M+\frac{3}{16 \pi a c} \frac{L}{T^{3}} \kappa \frac{\partial p}{\partial T}
$$

equations (5) for quasi-homologous transformations take form

$$
\begin{aligned}
\frac{d \eta^{1}}{d \rho}-\frac{d \xi}{r}= & -\frac{2 \xi}{d r}+\frac{\eta^{1}}{\rho}-\eta^{1} \frac{\partial}{\partial \rho}\left(\ln \frac{\partial p}{\partial \rho}\right)+\frac{\eta^{1}}{f}\left[\frac{3}{16 \pi a c} \frac{L}{T^{3}} \frac{\partial}{\partial \rho}\left(\kappa \frac{\partial p}{\partial T}\right)\right]-\frac{G \eta^{2}}{f} \\
& +\frac{\eta^{3}}{f}\left(\frac{3}{16 \pi a c} \frac{\kappa}{T^{3}} \frac{\partial p}{\partial T}\right)+\frac{\eta^{4}}{f}\left[-\frac{\partial}{\partial T}\left(\ln \frac{\partial p}{\partial \rho}\right)+\frac{3 L}{16 \pi a c} \frac{\partial}{\partial T}\left(\frac{\kappa}{T^{3}} \frac{\partial p}{\partial T}\right)\right] \\
\frac{d \eta^{2}}{d M}-\frac{d \xi}{d r}= & \frac{2 \xi}{r}+\frac{\eta^{1}}{\rho} \\
\frac{d \eta^{3}}{d L}-\frac{d \xi}{d r}= & \frac{2 \xi}{r}+\frac{\eta^{1}}{\rho}+\frac{\eta^{1}}{\rho} \frac{\partial \epsilon}{\partial \rho}+\frac{\eta^{4}}{\epsilon} \frac{\partial \epsilon}{\partial T} \\
\frac{d \eta^{4}}{d T}-\frac{d \xi}{d r}= & -\frac{2 \xi}{r}+\frac{\eta^{1}}{\rho}+\frac{\eta^{1}}{\kappa} \frac{\partial \kappa}{\partial \rho}+\frac{\eta^{3}}{L}+\eta^{4}\left(-\frac{3}{T}+\frac{1}{\kappa} \frac{\partial \kappa}{\partial T}\right) .
\end{aligned}
$$

Since the right-hand side of equation (15) depends only on $\rho$, therefore $\eta^{1}=\alpha_{1} \rho$, where $\alpha_{1}$ is constant. It is easy to verify, by the same argument and by substitution into equations (14) - (17), that also

$$
\eta^{2}=\alpha_{2} M, \quad \eta^{3}=\alpha_{3} L, \quad \xi=\frac{1}{3}\left(\alpha_{2}-\alpha_{1}\right) r
$$

where $\alpha_{2}, \alpha_{3}$ are constant. By substituting these equations into system (14)- (17) one obtains

$$
\begin{aligned}
& \left(\frac{d \eta^{4}}{d T}-\frac{2}{3} \alpha_{2}-\frac{4}{3} \alpha_{1}\right) \frac{\partial p}{\partial T}+\alpha_{1} \rho \frac{\partial^{2} p}{\partial \rho \partial T}+\eta^{4} \frac{\partial^{2} p}{\partial T^{2}}=0 \\
& -\frac{2}{3} \alpha_{2}-\frac{1}{3}+\alpha_{1} \rho-\frac{\partial}{\partial \rho}\left(\ln \frac{\partial p}{\partial \rho}\right)+\eta^{4} \ln \frac{\partial p}{\partial T}=0 \\
& \frac{4}{3} \alpha_{1}-\frac{1}{3} \alpha_{2}+\alpha_{3}-\frac{d \eta^{4}}{d T}+\alpha_{1} \frac{\rho}{\kappa} \frac{\partial \kappa}{\partial \rho}+\left(-\frac{3}{T}+\frac{1}{T} \frac{\partial \kappa}{\partial T}\right)=0 \\
& \left(\alpha_{3}-\alpha_{2}\right) \epsilon=\alpha_{1} \rho \frac{\partial \epsilon}{\partial T \rho}+\eta^{4} \frac{\partial \epsilon}{\partial T}
\end{aligned}
$$


Equations (20) and (21) imply that the opacity coefficient $\kappa$ and the energy generation rate $\epsilon$ are determined by the property of quasi-homologous temperature transformation generated by component $\eta^{4}(T) \partial / \partial T$. This gives us the following solutions

$$
\begin{aligned}
& \phi\left\{\rho \exp \left(-\alpha_{1} \int_{T_{0}}^{T} \frac{d t}{\eta^{4}}\right), \kappa \exp \left[\int_{T_{0}}^{T} \frac{d t}{\eta^{4}}\left(\frac{d \eta^{4}}{d t}-\frac{3 \eta^{4}}{t}+\frac{\alpha_{2}}{3}-\frac{4 \alpha_{1}}{3}-\alpha_{3}\right)\right]\right\}=0 \\
& \psi\left\{\rho \exp \left(-\alpha_{1} \int_{T_{0}}^{T} \frac{d t}{\eta^{4}(t)}\right), \rho \epsilon^{\alpha_{1} /\left(\alpha_{3}-\alpha_{2}\right)}\right\}=0
\end{aligned}
$$

where $\phi, \psi$ are arbitrary functions. Instead of form (22), equivalent and useful forms of representation of solutions (22) and (23) can be used in the further analysis [17, namely

$$
\begin{aligned}
& \kappa(\rho, T)=\exp \left[\int_{T_{0}}^{T} \frac{d t}{\eta^{4}}\left(\frac{d \eta^{4}}{d t}-\frac{3 \eta^{4}}{t}+\frac{\alpha_{2}}{3}-\frac{4 \alpha_{1}}{3}-\alpha_{3}\right)\right] f\left(\frac{1}{\rho} \exp \left(\alpha_{1} \int_{T_{0}}^{T} \frac{d t}{\eta^{4}}\right)\right) \\
& \epsilon(\rho, T)=\rho^{\left(\alpha_{3}-\alpha_{2}\right) / \alpha_{1}} g\left(\rho^{-\left(\alpha_{3}-\alpha_{2}\right) / \alpha_{1}} \exp \left(\left(\alpha_{3}-\alpha_{2}\right) \int_{T_{0}}^{T} \frac{d t}{\eta^{4}}\right)\right)
\end{aligned}
$$

where $f$ and $g$ are arbitrary functions.

It still remains to solve equations (18) and (19). Since we have some freedom in choosing the equation $\eta^{4}(T)$, and the function $p(\rho, T)$ is never precisely known, we look for those equations of state $p=p(\rho, T)$ that are enforced by the transformations generated by operator (2). It is easy to check by using equations (18) and (19) that $p=p(\rho, T)$ satisfies the continuity condition $\partial^{2} p / \partial T \partial \rho$ which in fact is a consistency condition for (18) and (19). Depending on the hyperbolic or parabolic character of equations (18) and (19) four cases can be distinguished. Let us discuss the solutions for these cases.

\subsection{Case I}

$$
X=\frac{\alpha_{2}-\alpha_{1}}{3} r \frac{\partial}{\partial r}+\alpha_{1} \rho \frac{\partial}{\partial \rho}+\alpha_{2} M \frac{\partial}{\partial M}+\alpha_{3} L \frac{\partial}{\partial L}+\eta^{4}(T) \frac{\partial}{\partial T} .
$$

The standard method of reduction to the canonical form, when applied to equation (19), gives

$$
\frac{\partial^{2} p(x, T)}{\partial x \partial T}=\frac{4 \alpha_{1} / 3+2 \alpha_{2} / 3}{\eta^{4}(T)} \frac{\partial p}{\partial x}
$$

where

$$
x=\rho \exp \left(-\alpha_{1} \int_{T_{0}}^{T} \frac{d t}{\eta^{4}(t)}\right) .
$$

In this case, the general solution of equation (26) assumes the form [17]

$$
p(x, T)=h(T)+\int_{x_{0}}^{x} g(\zeta) d \zeta \exp \left(\int_{T_{0}}^{T} \frac{4 \alpha_{1} / 3+2 \alpha_{2} / 3}{\eta^{4}(T)}\right)
$$

where $h(T)$ is of $C^{2}$ class and $g(\zeta)$ is of $C^{1}$ class of differentiability.

By substituting the general solution (27) in equation (18) we obtain additional condition for $h(T)$

$$
\eta^{4}(T) h^{\prime \prime}(T)+\left(\frac{4}{3} \alpha_{1}+\frac{2}{3} \alpha_{2}-\frac{d \eta^{4}}{d T}\right) h^{\prime}(T)=0 .
$$

The solution of this equation is

$$
h(T)=C_{1} \int^{T} \exp \left(\int^{\tau}\left(\frac{4}{3} \alpha_{1}+\frac{2}{3} \alpha_{2}\right) \frac{d t}{d \eta}\right) \frac{d \tau}{\eta^{4}(\tau)}+C_{2}
$$

where $C_{1}$ and $C_{2}$ are constants.

This solution implies that in equation (27) there is still the freedom in choosing a function $g=g(\zeta)$. 


\subsection{Case II}

$$
X=\frac{\alpha_{2}-\alpha_{1}}{3} r \frac{\partial}{\partial r}+\alpha_{2} M \frac{\partial}{\partial M}+\alpha_{3} L \frac{\partial}{\partial L}+\eta^{4}(T) \frac{\partial}{\partial T} .
$$

By proceeding in the same way as in the previous case we obtain

$$
p(\rho, T)=h(T)+\int^{\rho} g\left(\rho^{\prime}\right) \exp \left(\int^{T} \frac{2 \alpha_{2}}{3 \eta^{4}(t)} d t\right) d \rho^{\prime}
$$

where

$$
h(T)=C_{1} \int^{T} \exp \left(\frac{2}{3} \alpha_{2} \int^{\tau} \frac{d t}{\eta^{4}(t)}\right) \frac{d \tau}{\eta^{4}(\tau)}+C_{2}
$$

where $h, g$ satisfy differentiability conditions as in the previous case and $C_{1}, C_{2}$ are constants.

\subsection{Case III}

$$
X=\frac{\alpha_{2}-\alpha_{1}}{3} r \frac{\partial}{\partial r}+\alpha_{1} \rho \frac{\partial}{\partial \rho}+\alpha_{2} M \frac{\partial}{\partial M}+\alpha_{3} L \frac{\partial}{\partial L} .
$$

Similar calculations give

$$
p(\rho, T)=h(\rho)+\int^{T} \rho^{4 / 3+2 \alpha_{2} / 3 \alpha_{1}} g(t) d t
$$

where

$$
h(T)=C_{1} \rho^{4 / 3+2 \alpha_{2} / 3 \alpha_{1}}+C_{2}
$$

where $h, g$ satisfy the differentiability conditions as previously and $C_{1}, C_{2}$ are constants.

\subsection{Case IV}

$$
X=\frac{\alpha_{2}-\alpha_{1}}{3} r \frac{\partial}{\partial r}+\alpha_{2} M \frac{\partial}{\partial M}+\alpha_{3} L \frac{\partial}{\partial L} .
$$

In this case we have the following solutions

$$
p=p(\rho, T), \quad \alpha_{2}=0 .
$$

We can construct finite transformations and group invariants. In case I, for example, we obtain four independent invariants $L_{0}, M_{0}, r_{0}$, and $\rho_{0}$

$$
\begin{aligned}
L_{0}=L \exp \left(-\alpha_{3} \int^{T} \frac{d t}{\eta^{4}(t)}\right), & M_{0}=M \exp \left(-\alpha_{2} \int^{T} \frac{d t}{\eta^{4}(t)}\right), \\
r_{0}=r \exp \left(\frac{\alpha_{1}-\alpha_{2}}{3} \int^{T} \frac{d t}{\eta^{4}(t)}\right), & \rho_{0}=\rho \exp \left(-\alpha_{1} \int^{T} \frac{d t}{\eta^{4}(t)}\right) .
\end{aligned}
$$

By using these invariants, we can construct the new families of solutions, for instance

(i) if $L(T)$ is the solution of (12), (8) (9), and (10), then also

$$
L(E(T)) \exp \left(-\alpha_{3} \int_{E\left(T_{0}\right)}^{E(T)} \frac{d t}{\eta^{4}(t)}\right)
$$

is the solution, where $E(T)$ is a finite transformation of $T$, given from the solution $\bar{T}=E(T)$ of equation $d \bar{T} / d \tau=\eta^{4}(\bar{T})$ with the initial condition $\bar{T}(\tau=0)=T$.

(ii) if $M(T)$ is the solution of (12), (8) (9), and (10), then also

$$
M(E(T)) \exp \left(-\alpha_{2} \int_{E\left(T_{0}\right)}^{E(T)} \frac{d t}{\eta^{4}(t)}\right)
$$

is the solution. 


\section{Homologous symmetry transformations of structure equations with radiative transport}

It is well known that equations (12), (8) (9), and (10) admit similarity symmetries for the equation of state of ideal gas, $p \sim \rho T$. This fact induces a certain class of homologous solutions to the system. Some classical results were obtained by Stromgren [15].

In order to investigate how general our results are, let us assume the rescaling symmetry $\eta^{4}(T)=\alpha_{4} T$. Then, in Case I, for instance, the following equation of state is enforced

$$
p(x, T)=\frac{C_{1} T^{\left(4 \alpha_{1}+2 \alpha_{2}\right) / 3 \alpha_{4}}}{\frac{4}{3} \alpha_{1}+\frac{2}{3} \alpha_{2}+\alpha_{4}}+\int_{x_{0}}^{x} g(\zeta) d \zeta \frac{T^{1+\left(4 \alpha_{1}+2 \alpha_{2}\right) / 3 \alpha_{4}}}{\frac{4}{3} \alpha_{1}+\frac{2}{3} \alpha_{2}+\alpha_{4}} .
$$

One should notice that equation (36) contains the following form of the equation of state

$$
p=a \rho^{A}+b T^{B}+c \rho^{C} T^{D} .
$$

The infinitesimal operator corresponding to the homologous transformations takes the form

$$
X=\frac{\alpha_{2}-\alpha_{1}}{3} r \frac{\partial}{\partial r}+\alpha_{1} \rho \frac{\partial}{\partial \rho}+\alpha_{2} M \frac{\partial}{\partial M}+\alpha_{3} L \frac{\partial}{\partial L}+\eta^{4}(T) \frac{\partial}{\partial T} .
$$

The operator given by equation (37) has four independent invariants, for instance

$$
J_{1}=\rho r^{3 \alpha_{1} /\left(\alpha_{1}-\alpha_{2}\right)}, \quad J_{2}=\rho M^{-\alpha_{1} / \alpha_{2}}, \quad J_{3}=L M^{\alpha_{3} / \alpha_{2}}, \quad J_{4}=L T^{-\alpha_{3} / \alpha_{4}} .
$$

By using these invariants we can arrive at various homology theorems, for example the theorem associated with $J_{1}$ : if $\rho(r)$ is the solution of (12) and (8) - (10), then $\rho\left\{r \exp \left[\left(\alpha_{2}-\right.\right.\right.$ $\left.\left.\left.\alpha_{1}\right) / 3\right]\right\} \exp \left(-\alpha_{1}\right)$ is also the solution.

From $J_{3}$ we obtain $L \sim M^{\alpha_{3} / \alpha_{2}}$ that corresponds to the well known Eddington massluminosity dependence. Infinitesimal operator (37) generates a Lie algebra spanned by the basic operators

$$
X_{1}=-\frac{1}{3} r \frac{\partial}{\partial r}+\rho \frac{\partial}{\partial \rho}, \quad X_{2}=\frac{1}{3} r \frac{\partial}{\partial r}+M \frac{\partial}{\partial M}, \quad X_{3}=L \frac{\partial}{\partial L}, \quad X_{4}=T \frac{\partial}{\partial T} .
$$

We now briefly present the basis operators for some particular cases that are important from the physical point of view.

(i) Photon gas, $p \sim T^{4}$

$$
X_{1}=-\frac{r}{3} \frac{\partial}{\partial r}+\rho \frac{\partial}{\partial \rho}+\frac{T}{3} \frac{\partial}{\partial T}, \quad X_{2}=\frac{r}{3} \frac{\partial}{\partial r}+M \frac{\partial}{\partial M}+\frac{T}{6} \frac{\partial}{\partial T}, \quad X_{3}=L \frac{\partial}{\partial L}, \quad X_{4}=0 .
$$

(ii) Ideal gas, $p \sim \rho T$

$$
X_{1}=-\frac{r}{3} \frac{\partial}{\partial r}+\rho \frac{\partial}{\partial \rho}+\frac{T}{3} \frac{\partial}{\partial T}, \quad X_{2}=\frac{r}{3} \frac{\partial}{\partial r}+M \frac{\partial}{\partial M}+\frac{T}{6} \frac{\partial}{\partial T}, \quad X_{3}=L \frac{\partial}{\partial L}, \quad X_{4}=0 .
$$

(iii) Degenerate gas, $p \sim \rho^{5 / 3}$

$$
X_{1}=-\frac{r}{6} \frac{\partial}{\partial r}+\rho \frac{\partial}{\partial \rho}+\frac{M}{2} \frac{\partial}{\partial M}, \quad X_{2}=0, \quad X_{3}=L \frac{\partial}{\partial L}, \quad X_{4}=M \frac{\partial}{\partial M} .
$$

(iv) Relativistic degenerate electron gas, $p \sim \rho^{4 / 3}$

$$
X_{1}=-\frac{r}{3} \frac{\partial}{\partial r}+\rho \frac{\partial}{\partial \rho}, \quad X_{2}=L \frac{\partial}{\partial L}, \quad X_{3}=0, \quad X_{4}=M \frac{\partial}{\partial M} .
$$

(v) Ideal and photon gas

$$
X_{1}=-\frac{r}{3} \frac{\partial}{\partial r}+\rho \frac{\partial}{\partial \rho}+\frac{T}{3} \frac{\partial}{\partial T}, \quad X_{2}=0, \quad X_{3}=L \frac{\partial}{\partial L}, \quad X_{4}=0 .
$$


It is interesting to notice that the Eddington mass-luminosity dependence is not satisfied any longer, as there are no non-trivial invariants associated with $M$.

(vi) Ideal and degenerate gas

$$
X_{1}=-\frac{r}{6} \frac{\partial}{\partial r}+\rho \frac{\partial}{\partial \rho}+\frac{M}{2} \frac{\partial}{\partial M}+\frac{2 T}{3} \frac{\partial}{\partial T}, \quad X_{2}=0, \quad X_{3}=L \frac{\partial}{\partial L}, \quad X_{4}=0 .
$$

\section{Quasi-homologous stars with convective transport}

We start the analysis of stars with convection from equations (7)-(91) and (111) which describe the Newtonian static star with convective transport energy. The convective transport of energy means an exchange of energy between hotter and cooler layers through the exchange of macroscopic mass elements, the hotter of which move upwards while the cooler ones descend.

By analogy to the previous analysis instead of equation (11) it would be useful to operate the new equation that can be constructed if we assume the form of the equation of state $p=p(\rho, T)$. Then we obtain

$$
\frac{d \rho}{d r}=\left(\frac{\partial p}{\partial \rho}\right)^{-1}\left(-1+\Gamma(\rho, T) \frac{T}{p} \frac{\partial p}{\partial T}\right) \frac{G M \rho}{r^{2}}=f\left(\frac{\partial p}{\partial \rho}\right)^{-1} \frac{G M}{r^{2}} .
$$

We search for the symmetry operator in form (13) for system (7)-(9) and (11). After extension of operator (13) on the first derivatives we obtain the equations admissible for this operator $X^{\prime}$ in the form

$$
\begin{aligned}
\frac{d \eta^{1}(\rho)}{d \rho}-\frac{d \xi(r)}{d r}= & X\left(\ln f^{1}\right)=-\frac{2 \xi(r)}{r}+\frac{\eta^{1}(\rho)}{\rho}-\eta^{4} \frac{\partial}{\partial T} \ln \left(\frac{\partial p}{\partial \rho}\right)+\frac{\eta^{2}(M)}{M} \\
& -\eta^{1} \frac{\partial}{\partial \rho} \ln \left(\frac{\partial p}{\partial \rho}\right)+\frac{\eta^{1}(\rho)}{f}\left(\frac{T}{p} \frac{\partial p}{\partial T} \frac{\partial \Gamma}{\partial \rho}+\Gamma \frac{T}{p} \frac{\partial^{2} p}{\partial T \partial \rho}-\frac{\Gamma T}{p^{2}} \frac{\partial p}{\partial T} \frac{\partial p}{\partial \rho}\right) \\
& +\frac{\eta^{4}(T)}{f}\left[\frac{T}{p} \frac{\partial \Gamma}{\partial T} \frac{\partial p}{\partial T}+\frac{\Gamma}{p} \frac{\partial p}{\partial T}-\frac{\Gamma T}{p^{2}}\left(\frac{\partial p}{\partial T}\right)^{2}+\frac{\Gamma T}{p} \frac{\partial^{2} p}{\partial T^{2}}\right] \\
\frac{d \eta^{2}(M)}{d M}-\frac{d \xi(r)}{d r}= & X\left(\ln f^{2}\right)=\frac{2 \xi(r)}{r}+\frac{\eta^{1}(\rho)}{\rho} \\
\frac{d \eta^{3}(L)}{d L}-\frac{d \xi(r)}{d r}= & X\left(\ln f^{3}\right)=\frac{2 \xi(r)}{r}+\frac{\eta^{1}(\rho)}{\rho}+\frac{\eta^{1}}{\epsilon} \frac{\partial \epsilon}{\partial \rho}+\frac{\eta^{4}}{\epsilon} \frac{\partial \epsilon}{\partial T} \\
\frac{d \eta^{4}(T)}{d T}-\frac{d \xi(r)}{d r}= & X\left(\ln f^{4}\right)=-\frac{2 \xi(r)}{r}+\frac{\eta^{2}(M)}{M}+\frac{\eta^{1}}{\rho}+\frac{\eta^{1}}{\Gamma} \frac{\partial \Gamma}{\partial \rho}-\frac{\eta^{1}}{p} \frac{\partial p}{\partial \rho}+2 \frac{\eta^{4}}{\Gamma} \frac{\partial \Gamma}{\partial T} \\
& +\frac{\eta^{4}}{T}-\frac{\eta^{4}}{p} \frac{\partial p}{\partial T} .
\end{aligned}
$$

From equation (48) we obtain $\eta^{3}(L)=\alpha_{3} L$, where $\alpha_{3}$ is constant and equation (47) gives us the linear relation $\eta^{2}(M)=\alpha_{2} M+\beta_{2}$ and $\eta^{1}(\rho)=\alpha_{1} \rho$, where $\alpha_{1}, \alpha_{2}, \beta_{2}$ are constant. Then equation (48) determines the constraint equation in the form

$$
\frac{d \xi(r)}{d r}+\frac{2 \xi(r)}{r}=\alpha_{2}-\alpha_{1}
$$

which can be easily integrated and we obtain

$$
\xi(r)=\frac{\alpha_{2}-\alpha_{1}}{3} r+\frac{C}{r} .
$$

After substitution of the above solution to equation (46) and (49) we finally obtain that constant $C$ in (51) must vanish. 
Then from equation (48) we obtain

$$
\alpha_{1} \rho \frac{\partial \epsilon}{\partial \rho}+\eta^{4}(T) \frac{\partial \epsilon}{\partial T}-\left(\alpha_{3}-\alpha_{2}\right) \epsilon=0
$$

which can be integrated by using the standard characteristic method. The invariants are determined from the characteristic equation

$$
\frac{d \rho}{\alpha_{1} \rho}=\frac{d T}{\eta^{4}(T)}=\frac{d \epsilon}{\left(\alpha_{3}-\alpha_{2}\right) \epsilon}
$$

where we assume that $\alpha_{3}-\alpha_{2} \neq 0$ then

$$
C_{1}=\frac{\rho^{\left(\alpha_{3}-\alpha_{2}\right) / \alpha_{1}}}{\epsilon}, \quad C_{2}=\frac{\exp \left[\left(\alpha_{3}-\alpha_{2}\right) \int^{T} \frac{d T}{\eta^{4}(T)}\right]}{\epsilon} .
$$

It is well known that the general solution of equation (52) can be given in the form of any function $\psi$ of its invariants, i.e.

$$
\psi\left(\frac{\rho^{\left(\alpha_{3}-\alpha_{2}\right) / \alpha_{1}}}{\epsilon}, \frac{\exp \left[\left(\alpha_{3}-\alpha_{2}\right) \int^{T} \frac{d T}{\eta^{4}(T)}\right]}{\epsilon}\right)=0 .
$$

Following Sundman there is the equivalent form of the general solution in which the production energy function can be given in the exact form, namely

$$
\epsilon=\rho^{\left(\alpha_{3}-\alpha_{2}\right) / \alpha_{1}} g\left(\frac{\exp \left[\left(\alpha_{3}-\alpha_{2}\right) \int^{T} \frac{d T}{\eta^{4}(T)}\right]}{\rho^{\left(\alpha_{3}-\alpha_{2}\right) / \alpha_{1}}}\right) .
$$

From (46) or (49) we obtain that

$$
\eta^{3}(M)=\alpha_{2} M+\beta_{2}
$$

and $\beta_{2}=0$.

Therefore all components of operator $X$ except $\eta^{4}(T)$ are determined

$$
\xi(r)=\frac{\alpha_{2}-\alpha_{1}}{3} r, \quad \eta^{1}(\rho)=\alpha_{1} \rho, \quad \eta^{2}(M)=\alpha_{2} M, \quad \eta^{3}(L)=\alpha_{3} L .
$$

In our further analysis we assume that that $\Gamma=\Gamma_{0}=$ const. This assumption simplifies our calculations but we must remember that in general $\Gamma=d \ln T / d \ln p$. (If the energy transport is due to radiation and conduction then $\Gamma$ must be replaced by $\Gamma_{\text {rad }}=$ $3 \kappa L p / 16 \pi a c G m T^{4}$.) This property is destroyed if the material function, instead of being products of power $p$ and $T$ contains additive terms as in the general case with the equation of state. The simplest example is the addition of radiation to ideal gas such that $p=\mathcal{R} \rho T / \mu+a T^{4} / 3=p_{\mathrm{g}}+p_{\mathrm{r}}$. No strict homology (quasi-homology) relation is then possible. But one can try to make the enlarged system of equations in which the equation for $d \beta / d r$ is added $\left(\beta=p_{\mathrm{g}} / p\right)$. The corresponding equation is

$$
\frac{d \beta}{d r}=\beta \frac{d}{d r} \ln \left(\frac{\mathcal{R}}{\mu} \frac{\rho T}{p}\right)=\beta \frac{d}{d r} \ln \left(\frac{\rho \Gamma}{p(\rho, T)}\right) .
$$

Now $\beta$ is not constant and in equation (57) the relations determining $d \rho / d r, d T / d r$, and $d p / d r$ should be substituted. As a result we obtain that the enlarged system admits the homology relation.

Because we focus on the simplest possible case the problem with the constant $\Gamma$ is considered. For $\Gamma=\Gamma_{0}$, equation (46) assumes the form

$$
\begin{aligned}
\frac{\alpha_{2}-\alpha_{1}}{3}= & \alpha_{2}-\alpha_{1} \rho \frac{\partial}{\partial \rho} \ln \left(\frac{\partial p}{\partial \rho}\right)-\eta^{4} \frac{\partial}{\partial T}\left(\ln \frac{\partial p}{\partial \rho}\right)+\frac{\alpha_{1} \rho}{f}\left(\frac{\Gamma_{0} T}{p} \frac{\partial^{2} p}{\partial \rho \partial T}-\frac{\Gamma_{0} T}{p^{2}} \frac{\partial p}{\partial T} \frac{\partial p}{\partial \rho}\right) \\
& +\frac{\eta^{4}(T)}{f}\left[\frac{\Gamma_{0}}{p} \frac{\partial p}{\partial T}-\frac{\Gamma_{0} T}{p^{2}}\left(\frac{\partial p}{\partial T}\right)^{2}+\frac{\Gamma_{0} T}{p} \frac{\partial^{2} p}{\partial T^{2}}\right] .
\end{aligned}
$$


Quite similarly we can write equation (49) in the constant $\Gamma$ and we have

$$
\frac{d \eta^{4}(T)}{d T}-\frac{\alpha_{2}-\alpha_{1}}{3}=-\frac{2}{3}\left(\alpha_{2}-\alpha_{1}\right)+\alpha_{1}+\alpha_{2}+\frac{\eta^{4}(T)}{T}-\frac{\alpha_{1} \rho}{p} \frac{\partial p}{\partial \rho}
$$

or

$$
\alpha_{1} \rho \frac{\partial p}{\partial \rho}+\eta^{4}(T) \frac{\partial p}{\partial T}=\left(-\frac{d \eta^{4}}{d T}+\frac{\eta^{4}}{T}+\frac{4 \alpha_{1}+2 \alpha_{2}}{3}\right) p
$$

The solution of equation (59) can be given in the form of arbitrary function $\psi$ of its invariants, namely

$$
\psi\left(\rho^{-1} \exp \left(\int^{T} \frac{\alpha_{1}}{\eta^{4}} d t\right), p^{-1} \exp \left(\int^{T} \frac{d t}{\eta^{4}}\left(-\frac{d \eta^{4}}{d t}+\frac{\eta^{4}}{t}+\frac{4 \alpha_{1}+2 \alpha_{2}}{3}\right)\right)\right)=0
$$

or

$$
p(\rho, T)=\exp \left(\int^{T} \frac{d t}{\eta^{4}}\left(-\frac{d \eta^{4}}{d t}+\frac{\eta^{4}}{t}+\frac{4 \alpha_{1}+2 \alpha_{2}}{3}\right)\right) g\left(\rho^{-1} \exp \left(\int^{T} \frac{\alpha_{1}}{\eta^{4}} d t\right)\right) .
$$

After substitution the corresponding terms to (59) from the exact formula for $p(\rho, T)$, the following condition must be fulfilled

$$
\begin{aligned}
& \frac{d \eta_{4}}{d T}-\frac{\eta_{4}}{T}-\Gamma_{0} T\left(-\frac{d \eta_{4}}{d T}+\frac{\eta_{4}}{T}+\frac{4 \alpha_{1}+2 \alpha_{2}}{3}\right) \frac{1}{\eta_{4}} \frac{d \eta_{4}}{d T} \\
& -\Gamma_{0} T \eta_{4} \frac{\partial}{\partial T}\left[\left(-\frac{d \eta_{4}}{d T}+\frac{\eta_{4}}{T}+\frac{4 \alpha_{1}+2 \alpha_{2}}{3}\right) \frac{1}{\eta_{4}}\right]=0
\end{aligned}
$$

The above relation reduces to the simpler form

$$
\frac{d^{2} \eta_{4}}{d T^{2}}+\frac{1-\Gamma_{0}}{\Gamma_{0}} \frac{1}{T}\left(\frac{d \eta_{4}}{d T}-\eta_{4}\right)=0
$$

The solution of above equation for any $\Gamma_{0}$ can be obtained if we substitute $\eta_{4} \approx T^{x}$. Then

$$
x_{1}=1, \quad x_{2}=\frac{\Gamma_{0}}{\Gamma_{0}-1}
$$

and finally we

$$
\eta_{4}=C_{1} T+C_{2} T^{\left(\Gamma_{0}-1\right) / \Gamma_{0}}
$$

where $C_{1}$ and $C_{2}$ are constants.

Therefore, we find that for the general form of the equation of state the quasi-homology symmetry is admitted by the stellar structure, e.g. with convective transport, i.e., the operator of this symmetry is

$$
X=\frac{\alpha_{2}-\alpha_{1}}{3} r \frac{\partial}{\partial r}+\alpha_{1} \rho \frac{\partial}{\partial \rho}+\alpha_{2} M \frac{\partial}{\partial M}+\alpha_{3} L \frac{\partial}{\partial L}+C_{1} T+C_{2} T^{\left(\Gamma_{0}-1\right) / \Gamma_{0}} \frac{\partial}{\partial T}
$$

As a special case for $C_{2}=0$ we find that homologous symmetry is obvious and $p=$ $T^{\left(4 \alpha_{1}-2 \alpha_{2}\right) / 3 \alpha_{4}} g\left(\frac{T^{\alpha_{1} / \alpha_{4}}}{g}\right)$.

The non-trivial class of quasi-homologous (and homologous) relations can be obtained from the invariants. For example, from the invariant $J_{1}$ such that

$$
\frac{d L}{\alpha_{3} L}=\frac{d T}{C_{1} T+C_{2} T^{\left(\Gamma_{0}-1\right) / \Gamma_{0}}}, \quad J_{1}=L(T) \exp \left(-\alpha_{3} \int^{T} \frac{d \tau}{C_{1} \tau+C_{2} \tau^{\left(\Gamma_{0}-1\right) / \Gamma_{0}}}\right)
$$

it is possible to obtain a new class of solutions from the known ones which can be treated as a better reconstruction of the main sequence on the H-R diagram by using only theoretical assumptions. 


\section{Quasi-homologous stars with convective transport}

As the simplest example let us consider star filled by radiation, i.e., $p(\rho, T)=a T^{4}, \Gamma=\Gamma_{0}=$ $\frac{1}{4}, g=1$, then

$$
X=\frac{\alpha_{2}-\alpha_{1}}{3} r \frac{\partial}{\partial r}+\alpha_{1} \rho \frac{\partial}{\partial \rho}+\alpha_{2} M \frac{\partial}{\partial M}+\alpha_{3} L \frac{\partial}{\partial L}+\left(C_{1} T+C_{2} T^{-3}\right) \frac{\partial}{\partial T} .
$$

The important quasi-homologous theorem useful in the generation of a solution from the known ones can be formulated in the following way. If $L(T)$ is a solution of the stellar structure equation in a convective equilibrium, then

$$
L\left(T^{\prime}\right) \exp \left(-\alpha_{3} \int^{T^{\prime}} \frac{d \tau}{C_{1} \tau+C_{2} \tau^{-3}}\right)=\text { const }
$$

is also a solution, where $T^{\prime}=E(T)$ is a finite transformation which is determined as a solution of equation

and $T^{\prime}(\tau=0)=T$.

$$
\int^{T^{\prime}} \frac{d T}{C_{1} T+C_{2} T^{-3}}=\tau
$$

After integration we obtain the exact form of finite transformation

$$
T^{\prime}=E(T): C_{1}\left(T^{\prime}\right)^{4}+C_{2}=\left(C_{1} T^{4}+C_{2}\right) e^{4 C_{1} \tau} .
$$

This procedure can be repeated in the more general case of constant $\Gamma_{0}$. Then we obtain

$$
T^{\prime}=E(T): C_{1}\left(T^{\prime}\right)^{1 / \Gamma_{0}}+C_{2}=\left(C_{1} T^{1 / \Gamma_{0}}+C_{2}\right) e^{C_{1} \tau / \Gamma_{0}}
$$

\section{Conclusion}

We investigated symmetries for the equations of state of ideal gas, polytropic gas, photon gas, degenerated gas, as well as some mixed cases, and found the most general form of quasi-homologous transformation admitted by the system of equations describing Newtonian static stars. In considering the invariants of the symmetries group we noticed that they correspond to the relations describing physical characteristics of the stars, such as the Hertzsprung-Russell diagram or the mass-luminosity relation. The group invariants make possible constructing quasi-homologous theorems analogous to the Chandrasekhar homologous theorems, which provide a prescription for deriving new classes of solutions from the present known solutions.

We characterized, by computing infinitesimal operators, the structure of the group admissible by a system of equation describing Newtonian static stars in radiative and convective equilibrium. We have shown that, in the most general case, the equations admit an infinite parameter group of quasi-homologous transformations. These symmetries enforce appropriate equations of state. In the particular case of a five parameter homologous group of the Stromgren results are recovered.

The equations of state (36) and (61) and parameterization of energy production and capacity are very general. They contains both physical and non-physical situations. Our results suggest that group invariants have not always purely mathematical sense, but that their existence is closely associated with basic empirical facts such as the Hertzsprung-Russell diagram or the Eddington mass-luminosity dependence. The fact that the main sequence in the Hertzsprung-Russell diagram can be reconstructed from the invariants proved the effectiveness of the group analysis of fundamental equations of astrophysics. We indicated this in two examples on the the main sequence models and radiative and convective transport. The main result is that except of classical homology there is another type of quasi-homologous symmetry which can be applied to certain red giants (see [18, §32.2]).

Due to existence a new 'similarity' between different solutions, the quasi-homology relation offer qualitative but the helpful indication for interpreting or predicting the numerical solutions. 


\section{Acknowledgments}

The authors thanks dr Biesiada for allowing to use results originally going from our collaboration. We are very grateful dr Krawiec for help in preparing the paper and stimulating discussion.

\section{References}

[1] Chandrasekhar S 1957 An Introduction to the Study of Stellar Structure (New York: Dover Publ.)

[2] Rudzki A 1902 Bull. Astroph., 19134

[3] Eddington A S 1959 The Internal Constitution of the Stars (New York: Dover Publ.)

[4] Schwarzschild M 1958 Structure and Evolution of the Stars (Princeton: Princeton University Press)

[5] Biesiada M, Golda Z and Szydlowski M 1987 J. Phys. A 201313

[6] Biesiada M and Szydlowski M 1988 J. Phys. A: Math. Gen. 213409

[7] Golda Z and Szydlowski M 1999 Proceedings of the Eight Marcel Grossman Meeting on General Relativity Part A ed T Piran (Singapore: World Scientific) 330

[8] Ovsiannikov L V 1982 Group Analysis of Differential Equations (New York: Academic Press)

[9] Bluman G W and Kumei S 1989 Symmetries and Differential Equations (New York: Springer-Verlag)

[10] Stefani H 1989 Differential Equations: Their Solutions Using Symmetries (New York: Cambridge University Press)

[11] Olver P J 1993 Applications of Lie Groups to Differential Equations, 2nd ed. (New York: Springer-Verlag)

[12] Olver P J 1995 Equivalence, Invariants and Symmetry (New York: Cambridge University Press)

[13] Barenblatt G I 1996 Scaling, Self-similarity, and Intermediate Asymptotics (New York: Cambridge University Press)

[14] Collins C B 1977 J. Math. Phys. 181374

[15] Stromgren B 1937 Ery. Exapt. Naturw. 16465

[16] Bluman G W and Cole J D 1974 Similarity methods for differential equations (New York: Springer-Verlag)

[17] Sneddon I N 1957 Elements of Partial Differential Equations (New York: McGraw-Hill)

[18] Kippenhahn R and Weigert A 1990 Stellar Structure and Evolution (Berlin: SpringerVerlag) 